\title{
Dashboarding The Online Strategic Communications of Anti-slavery Organizations During COVID-19
}

\author{
Benjamin Lucas ${ }^{1}$, Liana Bravo-Balsa ${ }^{1}$, Vicky Brotherton ${ }^{1}$, Nicola \\ Wright $^{1}$, and Todd Landman ${ }^{1}$ \\ ${ }^{1}$ Rights Lab, University of Nottingham, United Kingdom
}

October 2020

\begin{abstract}
In this working paper, we investigate high-level changes in the online strategic communications of organizations engaged with SDG 8.7 (ending modern slavery) during the COVID-19 crisis. We present preliminary evidence of important semantic and thematic shifts based on data from Twitter during this time, with an emphasis on developing the SOLACE (Social Listening and Communications Engagement) dashboard, and with recommendations for important future research involving the use of social media data as a basis for distilling organizational-agenda proxies based on digital campaigns and activism during times of crisis.
\end{abstract}

\section{Introduction}

Online strategic communications (OSCs) are an important part of the broader communications arsenal for organizations addressing grand challenges, such as those articulated as part of the United Nations Sustainable Development Goals (SDGs). Leveraging the prominence of platforms including social media, and the rise of social media activism (see for example: Fisher, 2019), NGOs, government bodies, and various other civil society and activist actors make use of the wide-reach and ubiquitous nature of online communication to build support for their causes. This involves a mixture of carefully engineered campaigns, timely reactive commentary, and the re-sharing of, and participation in, the discussions of other actors on a real-time basis.

In times of crisis, both planned campaigns, longer-term activism and social movement development, and the general pace of ad-hoc discourse participation is thrown into disarray. In very simple terms, and phrased as a core question: how does an organization get the public to pay attention to an issue such as 
human exploitation (or for that matter: climate change, poverty, and hunger, among others), when the world's media and social media is overwhelmed with a global pandemic crisis?

To begin to answer this question, we present an initial overview of the SOLACE (Social Listening and Communications Engagement) dashboard, developed using the $\mathrm{R}$ ecosystem. The aim here is to collect, distill and present information related to high-level thematic and semantic changes in the OSCs of organizations engaged with SDG 8.7 (ending modern slavery) during the COVID-19 crisis. We do so against a backdrop of using visualization tools to support faster insight generation and decision making around (1) anti-slavery legislation (e.g. Allain and Schwarz, 2020), (2) COVID-19 in general (e.g. Lucas et al., 2020; Rufai and Bruce, 2020), and, (3) other major societal challenges (Nica-Avram et al., 2020), as well as recent developments in the deployment of novel data analytics approaches within human rights research (e.g. Landman, 2020).

\section{Method and Dashboard Demonstration}

To develop SOLACE, first, we collected data using the $\mathrm{R}$ package 'rtweet' (Kearney et al., 2020) to retrieve all available Tweets and Retweets from a precompiled list of the most Twitter-active anti-slavery organizations from around the world via interfacing with the public Twitter API. For this demonstration, we use a dataset comprising 30001 Tweets and 16231 Retweets made by these organizations between the 1st of January 2020 and the 31st of August 2020 (244 days in total).

After data manipulation (using a collection of ancillary packages), we used a series of group-by functions to construct counts for a selection of terms used within Tweets and Retweets (showing the usage of focal terms as a percentage of all daily Tweets / Retweets). We used the R package ggplot2 (Wickham et al., 2020) to create base visualizations, and then plotly (Sievert et al., 2020) for dashboarding. Figure 1 shows an overview of the Tweets by our focal organizations mentioning Coronavirus and COVID-19. This was achieved by filtering and counting Tweets containing the term "coronavirus_" or the stem "covid_" (either in text or in Hashtags). One notable peak in activity was on May 2nd, where $43.14 \%$ of all Tweets made by our focal organizations contained "covid_".

More specifically, the term "coronavirus_" began showing up in Tweets at the end of January, but it was not until mid-March that it began to be widely used. It reached its peak on April 18th. Tweets containing the word "covid_" began to appear almost a month later, in the second half of February, but by the end of March it was more widely used than "coronavirus_", to the extent of almost replacing it entirely - a trend that has continued to date. This is likely partly reflective of the development and evolution of naming conventions 
earlier on in the pandemic (see for example: World Health Organization, 2020).

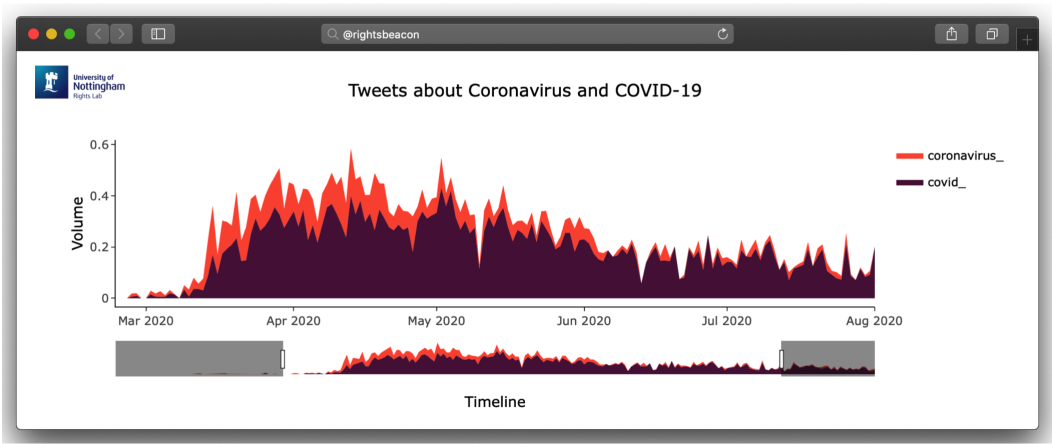

Figure 1: Coronavirus and COVID-19 Usage

As demonstrated in Figure 1, using the plotly engine allows us to first customize visualizations (in ggplot2), and then dashboard them, using interactive navigation functionality provided within the package, and exportable in html format for web hosting. Here, we have added a navigation bar, to allow users to change the viewing resolution down to a daily level (by selecting time windows of interest).
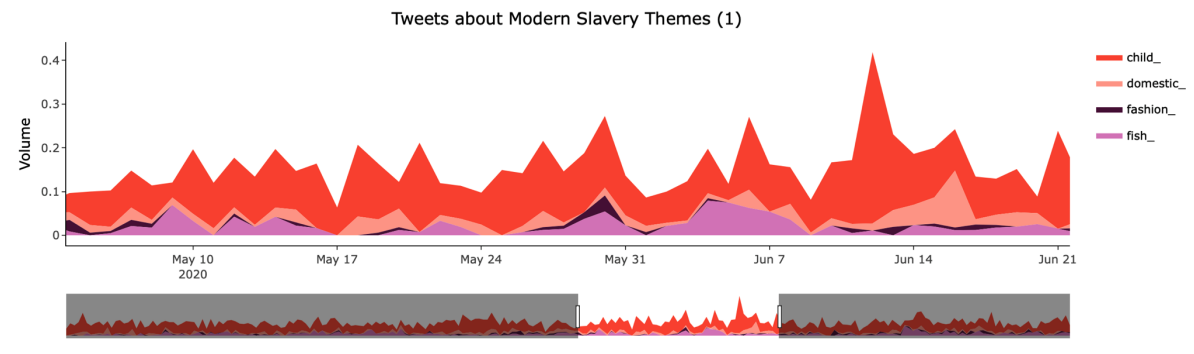

Timeline

Figure 2: Anti-slavery Themes within Tweets during COVID-19 (1)

Figures 2 and 3 capture core anti-slavery themes within Tweets, representing the strategic communication agendas of focal organizations (Figure 3 also shows an example of optional hover labels added to the navigation functionality). We make use of word stems to provide a simple overview of the thematic content within a Tweet. For this demonstration, these are grouped alphabetically, with Figure 2 showing "child_", "domestic_", "fashion_" and "fish_", capturing issues related to child exploitation, domestic violence, domestic abuse and domestic 
slavery, exploitation in the garment industry, and exploitation in the fishing industry (the latter two being common industrial case studies within the antislavery sphere), and Figure 3 showing "homeless_", "migrat_", "refugee_" and "sex_", capturing issues linking homelessness and modern slavery, migration and immigration, and its links to modern slavery, and relatedly, refugees and issues around displaced persons and modern slavery, and finally, issues related to sexual exploitation and sex trafficking.

More specifically, our focal anti-slavery organisations have (based on this dataset), focused more on communicating issues related to children (e.g. exploitation) than on domestic abuse or the fishing and garment industry cases. From January to September, Tweets mentioning the word stem "child_" have been considerably more prevalent than those that mention the word stems "domestic_", "fashion_" and "fish_". Tweets containing "domestic_" only surpassed those containing 'child' on June 16th, possibly coinciding with a debate in the House of Commons on the Domestic Abuse Bill 2019-21 in the UK. Meanwhile, "fashion_" overtook "child_" on July 5th, the same day the Leicester fast-fashion scandal broke. The peak in the usage of the word stem "refugee_" corresponds with World Refugee Day (June 20th). Tweets containing "refugee_" were more prevalent than those containing "migrat.", "sex_" or "homeless_". This trend became even more pronounced from mid-March onwards.

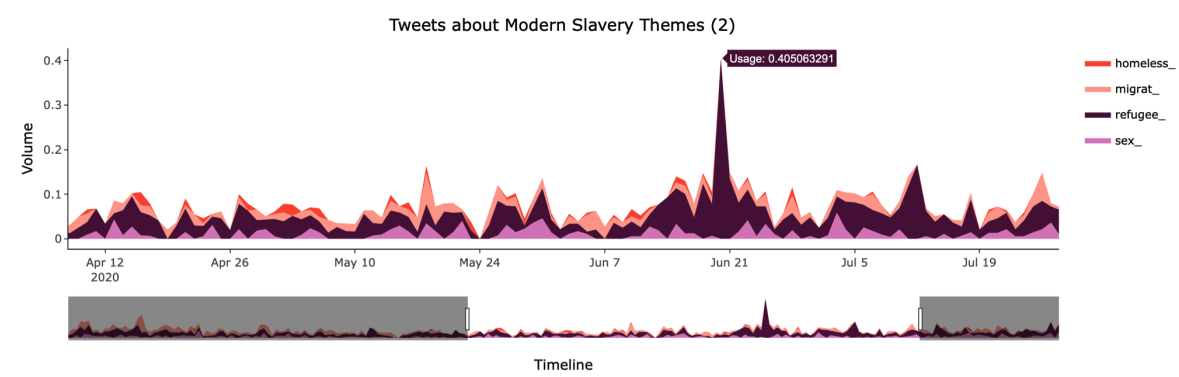

Figure 3: Anti-slavery Themes within Tweets during COVID-19 (2)

Our final example (Figures 4 and 5) uses word stems to capture what Lucas and Landman (2020) call semantic versus thematic capture in the context of anti-slavery strategic communications. Here, we use the stems "risk.", "surviv_", "victim_", and "vulnerab_" to capture common anti-slavery parlance (see: Nicholson et al., 2018), and emergent trends in terms of shifts in language tense and issue framing brought about by the COVID-19 crisis (e.g. possible shifts from discussions around victims and survivors of modern slavery toward communications around vulnerable populations). Figure 4 also demonstrates the use of interactive functionality to zoom in on daily resolution, while Figure 5 shows a comparison between two different higher-resolution time windows. 


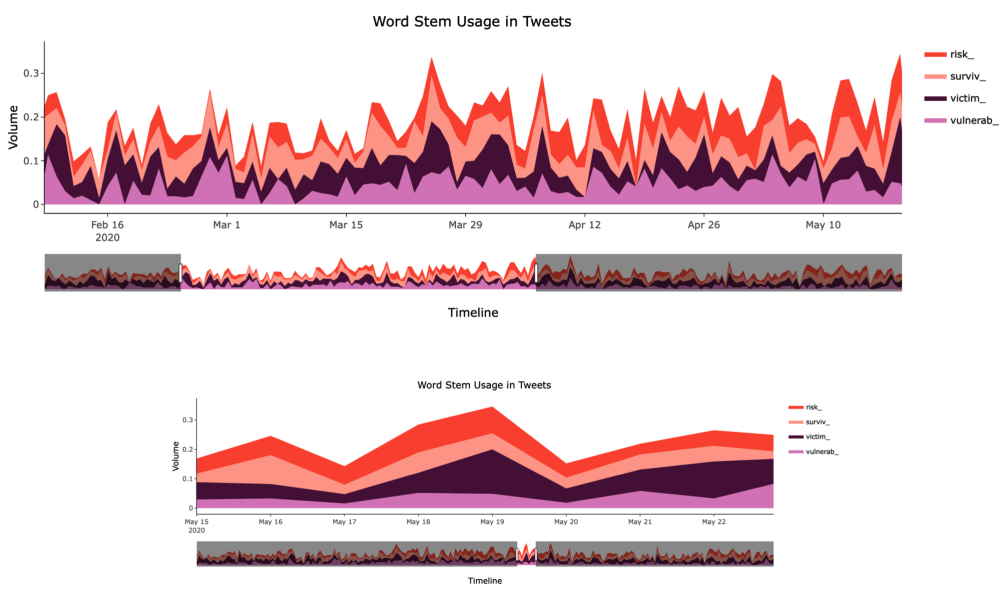

Figure 4: Key Anti-slavery Word Stems within Tweets during COVID-19
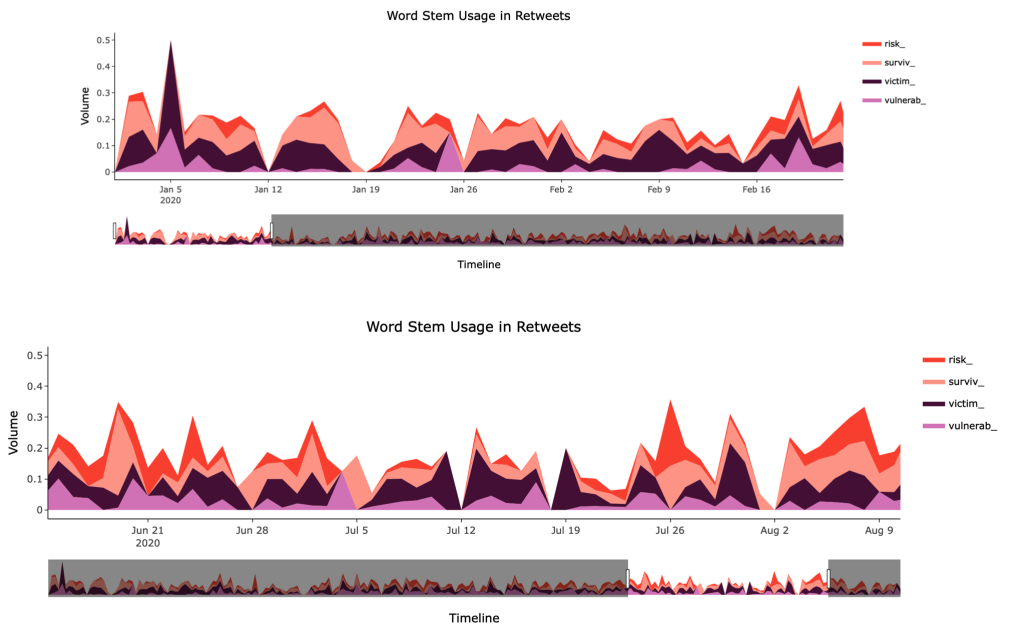

Figure 5: Key Anti-slavery Word Stems within Retweets during COVID-19

\section{Trend Distillation}

Figures 6 and 7 show semantic word stems distilled into curvilinear trend lines using LOESS smoothing in ggplot2. The $y$ axis once again shows usage as percentages. Specifically, these figures serve to illustrate and emphasize the fact that although the interactive raw data visualizations presented above provide useful insights (e.g. in terms of allowing researchers to draw their own conclusions and to zoom in on specific days and weeks, guided by particular 
news events and high-level trends), it is important to look at other levels of resolution and different noise handling approaches to establish patterns of activity. Here, usage (in percentage terms) varies considerably between word stems (representing semantic focus), and between Tweet and Retweet content.

For example, the word stems displayed here for Tweets: "risk_" $(4.15 \% \pm 2.35 \%)$, "surviv_" $(4.66 \% \pm 2.58 \%)$, "victim_" $(5.47 \% \pm 2.90 \%)$, "vulnerab_" $(3.59 \% \pm 2.49 \%)$, and Retweets: "risk_" $(3.22 \% \pm 3.24 \%)$, "surviv_" $(4.30 \% \pm 3.94 \%)$, "victim_" $(5.04 \% \pm 4.40 \%)$, "vulnerab_" $(3.07 \% \pm 3.30 \%)$, do not exhibit major differences between word stems and content types (Tweets and Retweets) at the most basic statistical levels, although the simple transformations used in Figures 6 and 7 do indeed reveal distinct patterns, when taken with reference to the evolution of the COVID-19 crisis.

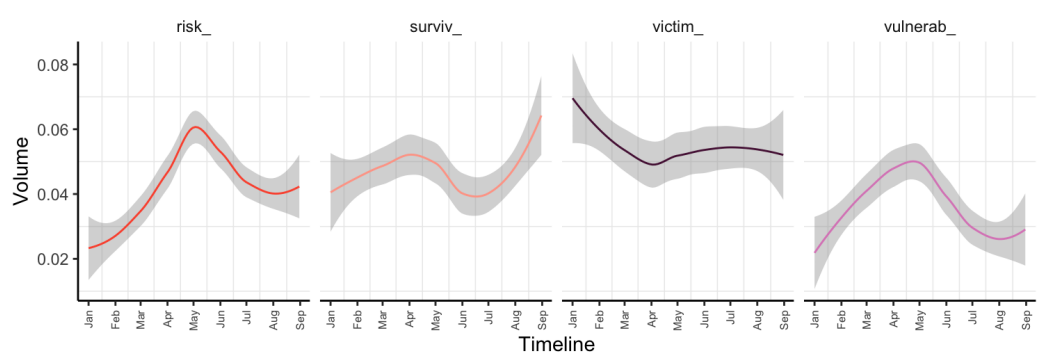

Figure 6: Summary of Semantic Trends from Tweets

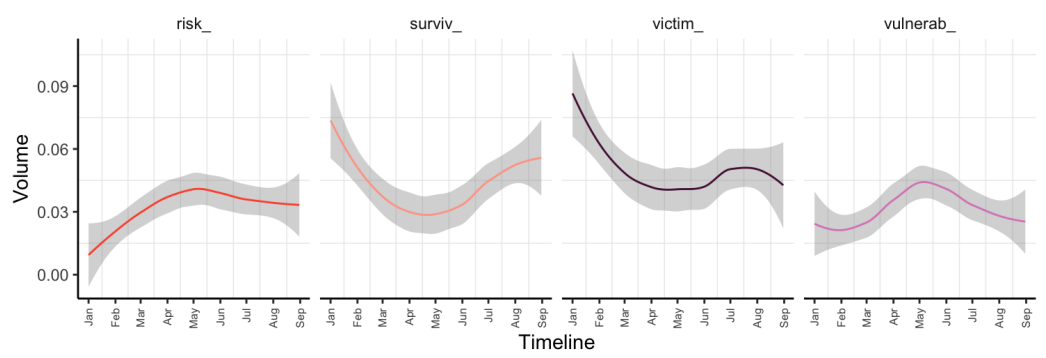

Figure 7: Summary of Semantic Trends from Retweets 


\section{Concluding Remarks}

This working paper demonstrates a working prototype of our proposed $\mathrm{SO}$ $L A C E$ dashboard, focusing on high-level changes in the OSCs of organizations engaged with SDG 8.7 (ending modern slavery) during the COVID-19 crisis. The main takeaways are as follows:

(1) Granular monitoring and data visualization, as facilitated by dashboard tools is a key part of building future multi-disciplinary understanding around complex social phenomena such as online mass-communication, digital activism and digital campaign management. Toolkits such as the one presented here, allow decision makers (e.g. activists, policy makers) to zoom in on the timelines of specific events, such as those triggered by news media and public discourse developments, to understand and draw their own conclusions about how a given issue unfolds, in terms of (a) the changing priorities and agendas (based on campaign and messaging proxies) of organizations involved, and (b) the most relevant and effective semantic terms being used to frame and present a given issue, with the collective goal being, (c) to maximize salience and engagement around as wider spectrum of relevant stakeholders as possible. Agenda analysis in this format will also involve filtering out the noise of COVID-19 as a dominant headline, to establish meaningful patterns of activity reflecting substantive agenda shifts and the assessment and forecasting of present and ongoing risks respectively - including sector-specific concerns (both policy and industrial).

(2) More specifically, whilst terms such as 'social listening' often refer to the monitoring of public discussion - the tracking of organization-side strategic communications at scale offers an important avenue for future research and practice development along the lines of thematic and semantic engineering. Specifically, dashboard tools should capture the engineering process of messages that are both timely (in thematic terms), and salient (in semantic terms). This means capturing key topics highlighted by the news media and the engagement of the general public, as well as developing a 'tone' that best communicates current priorities and urgencies. Issues for anti-slavery organizations to avoid via their OSCs in the current climate include becoming 'lost' in the wave (or aforementioned noise) of COVID-19 related messaging and 'compassion fatigue' (see: Maier, 2015).

(3) Future extensions of our work will include dashboarding \#Hashtags and @Mentions to present information around user-indexed themes, and the often complex networks of actors involved in public discussion (across different geographic areas) respectively. Simple statistical overviews of metrics such as participation in \#Hashtags and the volume of Tweet and Retweet activity from specific organizations (and associated engagement metrics) are important in and of themselves. Future work will also extend into dashboarding other relevant Twitter-streams within the SDG 8.7 sphere (e.g. law 
enforcement, grass-roots activism and collective action). Importantly, future research will also use the general dashboarding template presented here to advance discovery along the lines of thematic and semantic extraction, by employing methods such as sentiment dynamics extraction and topic modeling (see for example: Kim et al., 2016 and Stokes et al., 2020) within the apparatus.

(4) Perhaps the most important opportunity for extension arising from this working paper involves the formal operationalization of frameworks, such as that proposed in Gardner et al. (2020) (i.e. where vocabulary from datastreams such as social media is mapped to expert-defined dimensions of a given anti-slavery combat framework or anti-slavery agenda). Other questions can also be asked of the information generated by our agenda-proxy-focus approach, such as the extent to which relevant anti-slavery actors are engaging in proactive versus reactive agenda and campaign formulation, any issues around lag and latency, and the extent to which communication in strategic communication and campaign formats is consistent or divergent with other paradigms (e.g. especially the frontline survivor narrative approach - see: Nicholson et al., 2018). Another central question here is that of issue displacement, where core anti-slavery themes and emergent topics are replaced by newer and more salient issues over time.

In conclusion, we hope this work is seen as an important, complementary addition to the broader remote sensing (see: Jackson and Lucas, 2020), social movement, and strategic communications perspectives emergent in modern slavery scholarship. 


\section{References}

Allain, J. and Schwarz, K., 2020. Antislavery in Domestic Legislation World Mapping Visualisation Toolkit. [Link]

Fisher, D.R., 2019. The Broader Importance of \#FridaysForFuture. Nature Climate Change, 9(6), pp.430-431.

Gardner, A., Northall, P. and Brewster, B., 2020. Building Slavery-free Communities: A Resilience Framework. Journal of Human Trafficking, pp.1-16.

Jackson, B. and Lucas, B., 2020. A COVID-19 Response to Modern Slavery using AI Research. Delta 8.7 Forum. [Link]

Kearney, M.W., Heiss, A. and Briatte, F., 2020. R Package 'rtweet'. [Link]

Kim, E.H.J., Jeong, Y.K., Kim, Y., Kang, K.Y. and Song, M., 2016. Topic-based content and sentiment analysis of Ebola virus on Twitter and in the news. Journal of Information Science, 42(6), pp.763-781.

Landman, T., 2020. Measuring Modern Slavery: Law, Human Rights, and New Forms of Data. Human Rights Quarterly, 42(2), pp.303-331.

Lucas, B., Elliot, B. and Landman, T., 2020. Online Information Search During COVID-19. arXiv preprint arXiv:2004.07183.

Lucas, B. and Landman, T., 2020. Social Listening, Modern Slavery and COVID-19. Rights Lab Working Paper.

Maier, S.R., 2015. Compassion fatigue and the elusive quest for journalistic impact: A content and reader-metrics analysis assessing audience response. Journalism Mass Communication Quarterly, 92(3), pp.700-722.

Nica-Avram, G., Harvey, J., Goulding, J., Lucas, B., Smith, A., Smith, G. and Perrat, B., 2020, FIMS: Identifying, Predicting and Visualising Food Insecurity. In Companion Proceedings of The Web Conference 2020 (pp. 190-193).

Nicholson, A., Dang, M. and Trodd, Z., 2018. A Full Freedom: Contemporary Survivors' Definitions of Slavery. Human Rights Law Review, 18(4), pp.689-704.

Rufai, S.R. and Bunce, C., 2020. World Leaders' Usage of Twitter in Response to the COVID-19 Pandemic: A Content Analysis. Journal of Public Health, 42(3), pp.510-516,

Sievert., C. Parmer, C., Hocking, T., Chamberlain, S., Ram, K., Corvellec, M., Despouy, P., and Plotly Technologies Inc., 2020. R package plotly. [Link]

Stokes, D.C., Andy, A., Guntuku, S.C., Ungar, L.H. and Merchant, R.M., 2020. Public Priorities and Concerns Regarding COVID-19 in an Online Discussion Forum: Longitudinal Topic Modeling. Journal of General Internal Medicine, 35(7), pp.2244-2247.

Wickham, H., Chang, W., Henry, L., Pedersen, T.L., Takahashi, K., Wilke, C., Woo, K., Yutani, H., Dunnington, D. and RStudio. 2020., R package ggplot2. [Link]

World Health Organization., 2020. Naming the Coronavirus Disease (COVID-19) and the Virus that Causes it. [[Link] 